\title{
GRAVITATIONAL DEVIATION REACTION
}

TERRENCE J. SEJNOWSK I

University of California at Santa Barbara, Calif., U.S.A.

(Presented by J. B. Hartle)

\begin{abstract}
A gravitational radiation reaction tensor is calculated for a point quadrupole source in the fast motion approximation. The calculation is in direct analogy with Dirac's original calculation of electromagnetic radiation reaction. In the gravitational case a tidal deviation reaction tensor results which is linear in the fifth derivatives of the source quadrupole moments.
\end{abstract}

